\title{
The neural circuitry supporting goal maintenance during cognitive control: a comparison of expectancy AX-CPT and dot probe expectancy paradigms
}

\author{
Pilar Lopez-Garcia ${ }^{1,2,3}$ - Tyler A. Lesh ${ }^{3}$. Taylor Salo ${ }^{3}$ - Deanna M. Barch ${ }^{4}$. \\ Angus W. MacDonald III ${ }^{5}$. James M. Gold ${ }^{6}$. J. Daniel Ragland ${ }^{3} \cdot$ Milton Strauss $^{7}$. \\ Steven M. Silverstein ${ }^{8}$. Cameron S. Carter ${ }^{3}$
}

Published online: 22 October 2015

(C) Psychonomic Society, Inc. 2015

\begin{abstract}
Goal maintenance is an aspect of cognitive control that has been identified as critical for understanding psychopathology according to criteria of the NIMH-sponsored CNTRICS (Cognitive Neuroscience Treatment Research to Improve Cognition in Schizophrenia) and Research Domain Criteria (RDoC) initiatives. CNTRICS proposed the expectancy AX-CPT, and its visual-spatial parallel the dot probe expectancy (DPX), as valid measures of the cognitive and neural processes thought to be relevant for goal maintenance. The goal of this study was to specifically examine the functional neural correlates and connectivity patterns of both goal maintenance tasks in the same subset of subjects to further validate their neural construct validity and clarify our understanding of the nature and function of the neural circuitry engaged by the tasks. Twenty-six healthy control subjects performed both the letter (AX) and dot pattern (DPX) variants of the CPT during fMRI. Behavioral performance was similar between tasks. The 2 tasks engaged the same brain networks including dorsolateral prefrontal cortex (DLPFC) and dorsal parietal regions, supporting their validity as complementary measures
\end{abstract}

Pilar Lopez-Garcia

p.lopez@uam.es

\footnotetext{
Universidad Autonoma de Madrid, Madrid, Spain

CIBERSAM, Madrid, Spain

University of California at Davis, Davis, CA, USA

Washington University in St Louis, St Louis, MO, USA

University of Minnesota, Minneapolis, MN, USA

6 University of Maryland, College Park, MD, USA

7 Case Western Reserve University, Cleveland, OH, USA

8 Rutgers University, New Brunswick, NJ, USA
}

of the goal maintenance construct. Interestingly there was greater engagement of the frontal opercular insula region during the expectancy AX-CPT (letter) and greater functional connectivity between the PFC and medial temporal lobe in the DPX (dot pattern). These differences are consistent with differential recruitment of phonological and visual-spatial processes by the two tasks and suggest that additional long-term memory systems may be engaged by the dot probe version.

Keywords Goal maintenance · Context processing · Cognitive control $\cdot$ Connectivity

The need to establish valid, sensitive, and reliable measures of specific cognitive functions and their neural substrates has been highlighted by two initiatives in the field of psychopathology research. The first was the CNTRICS (Cognitive Neuroscience Treatment Research to Improve Cognition in Schizophrenia) initiative, which was conceived to identify cognitive constructs linked to specific brain networks that were selectively impaired in schizophrenia, and to identify tools that effectively measured those cognitive constructs that could be optimized for use in clinical settings (Barch et al., 2009; Carter \& Barch, 2007; Carter et al., 2008). The second was the Research Domain Criteria Initiative (RDoC), which was conceived to identify key domains of behavioral and biological processes involved in both normal functioning and psychopathology, especially those that can account for signs, symptoms, and behaviors independent of diagnostic category. One of the initial five RDoC domains was Cognitive Systems, with a number of the targeted constructs overlapping those identified by CNTRICS.

Goal maintenance was one of the aspects of cognition identified by CNTRICS as ready for immediate translational study in schizophrenia (Barch et al., 2009) and identified by RDoC 
as one of the core components of Cognitive Systems. Goal maintenance refers to the processes involved in the representation and active maintenance of goals or rules based on internal or external cues to produce appropriate behavioral responses (Barch \& Smith, 2008; Servan-Schreiber, Cohen, \& Steingard, 1996). The construct of goal maintenance has been linked with the activation of dorsolateral regions of the prefrontal cortex (DLPFC) in several studies (Barch et al., 1997; Barch et al., 2001; Holmes et al., 2005; Lesh et al., 2013; MacDonald \& Carter, 2003; MacDonald, Pogue-Geile, Johnson, \& Carter, 2003; MacDonald, Carter, et al., 2005; Paxton et al., 2008; Perlstein, Carter, Noll, \& Cohen, 2001), in particular with Brodmann Areas 46 and 9. This region seems to be crucial for the representation and maintenance of goal-related information that is important to guide behavior.

Goal maintenance is a cognitive construct repeatedly shown to be altered in schizophrenia (Barch et al., 2001; Barch \& Braver, 2005; Braver, Barch, \& Cohen, 1999; Braver \& Cohen, 1999; Cohen \& Servan-Schreiber, 1993). It has been suggested that a range of cognitive deficits in schizophrenia may reflect disturbances in the ability to represent and maintain goal information due to disturbed DLPFC function (Barch et al., 2001; Holmes et al., 2005; MacDonald, Carter, et al., 2005). Furthermore, goal maintenance impairments in schizophrenia could contribute to patients' deficits in working memory, attention, and executive functioning (Jones, Sponheim, \& MacDonald, 2010). Deficits in goal maintenance have been reported in chronic and first-episode patients (Barch, Carter, MacDonald, Braver, \& Cohen, 2003; Cohen et al., 1999; Javitt, Shelley, Silipo, \& Lieberman, 2000), and in their healthy siblings (MacDonald et al., 2003) and are an early predictor of risk for psychosis (Niendam et al., 2014).

Two of the tasks proposed by CNTRICS to assess goal maintenance were the expectancy AX-CPT and DPX (for a review, see MacDonald, 2008). Cohen and colleagues (Cohen et al., 1999; Servan-Schreiber et al., 1996) originally developed the expectancy AX task, a version of the classic Continuous Performance Test (CPT; Rosvold, Mirsky, Sarason, Bransome, \& Beck, 1956). In the expectancy AXCPT, subjects are presented with a series of cues and probes (AX, AY, BX, and BY trials), and a target response has to be made when an X probe follows an A cue; any other pattern would be a nontarget response. What makes the expectancy version different from the classic CPT is a high proportion of AX trials, which creates two prepotent tendencies to the A-cue trials. The first is the tendency to give a target response to the $\mathrm{X}$, since the majority of the trials are $\mathrm{AX}$ trials. When the $\mathrm{X}$ is not preceded by an A (B-cue trials), the goal information provided by the cue needs to be used to inhibit the prepotent tendency to respond target to the $\mathrm{X}$. The second is the tendency to prepare to respond to probes following an $\mathrm{A}$ cue. If the $\mathrm{A}$ cue is not followed by an $\mathrm{X}$ (referred to as AY trials), then individuals who have been using the information provided by the cue to prepare their response either make more errors or are slowed as they overcome the prepotency engendered by the A cues. Thus, the information represented by the cue is critical to make a correct target or nontarget response. Subjects with good goal maintenance show few errors on AX and BX trials but tend to make errors on $\mathrm{AY}$ trials. In contrast, participants with poor goal maintenance show errors on $\mathrm{AX}$ on $\mathrm{BX}$ trials but do relatively well on $\mathrm{AY}$ trials.

The dot pattern expectancy task (DPX) was developed as a variant of the expectancy AX-CPT, to overcome time constraints and ceiling effects associated with the AX-CPT, particularly in nonclinical populations. The DPX uses dot patterns instead of letters (see Fig. 1). In the expectancy AX task, relatively long interstimuli intervals (ISIs) are required as the letters are easily maintained, whereas novel patterns degrade more quickly in storage (MacDonald, Goghari, et al., 2005). Moreover, the dot pattern configurations increase the difficulty of the AY trials for healthy participants, overcoming ceiling effects and increasing the discriminating power for this trial type. Both tasks (AX and DPX) have been shown to have equivalent abilities to measure individual differences in goal maintenance (MacDonald, Goghari, et al., 2005). Confirmatory factor analyses have shown that the AX and BX conditions of both the AX and DPX tasks measure a common latent construct (MacDonald, Goghari, et al., 2005). Furthermore, both the AX-CPT and the DPX are sensitive to context processing deficits in schizophrenia patients and their siblings (Henderson et al., 2012; Lopez-Garcia et al., 2013; MacDonald, Goghari, et al., 2005; Zhang et al., 2012; Zhu et al., 2013).

Central to the issue of measuring specific deficits in schizophrenia and other disorders is the construct validity of the measures. Functional MRI is a powerful tool for establishing, at the neural systems level, the construct validity of a given measure as well as for dissecting the component processes and their associated neural systems to comprise a broader overarching cognitive construct - in the present case, goal maintenance. The expectancy AX-CPT has been used in numerous imaging studies (Barch et al., 1997; Barch et al., 2001; Holmes et al., 2005; MacDonald \& Carter, 2003; MacDonald, Carter, et al., 2005; Paxton et al., 2008; Perlstein et al., 2001) and has shown both the role of DLPFC in goal maintenance and evidence for altered DLPFC activity associated with impaired goal maintenance in schizophrenia. This study, in which we compare brain activity between the DPX and AX-CPT, is the first fMRI study of the DPX. The goal of this study was to test the hypothesis that both tasks would engage the canonical fronto-parietal cognitive control network, reflecting their neural construct validity as measures of cognitive control. Additionally, we sought to examine whether the differences in stimulus characteristics in the two tasks (expectancy AX uses letters which would lead subjects to engage phonological processes, the 


\section{AX-CPT Parameters and Timing}

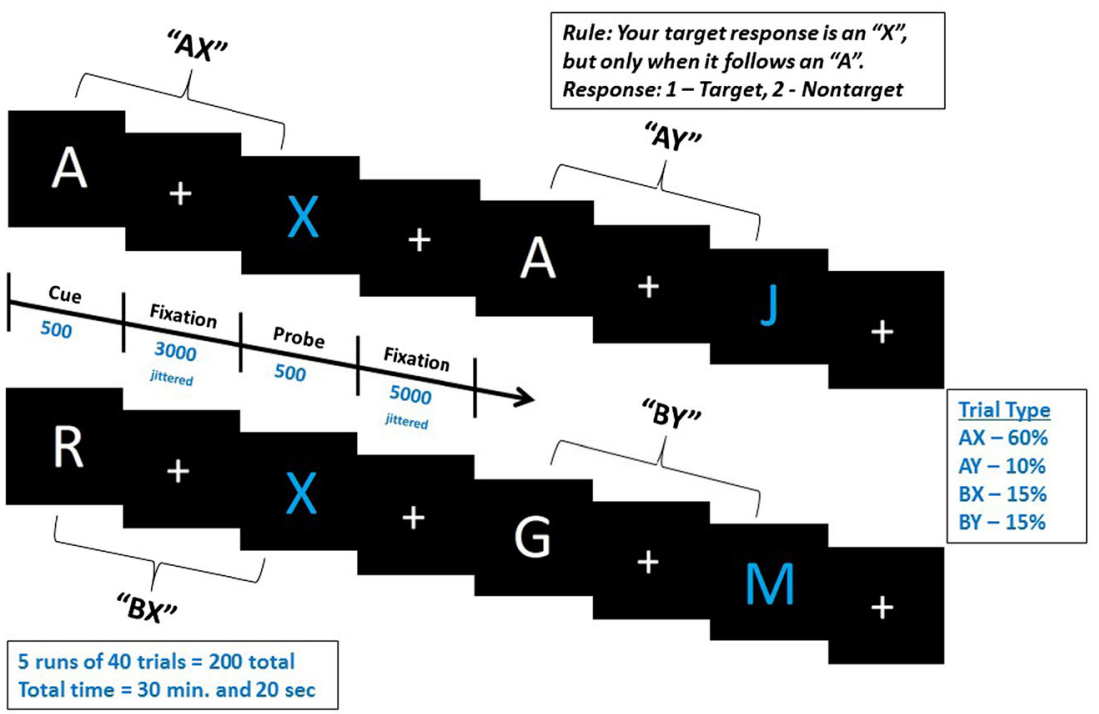

DPX Parameters and Timing

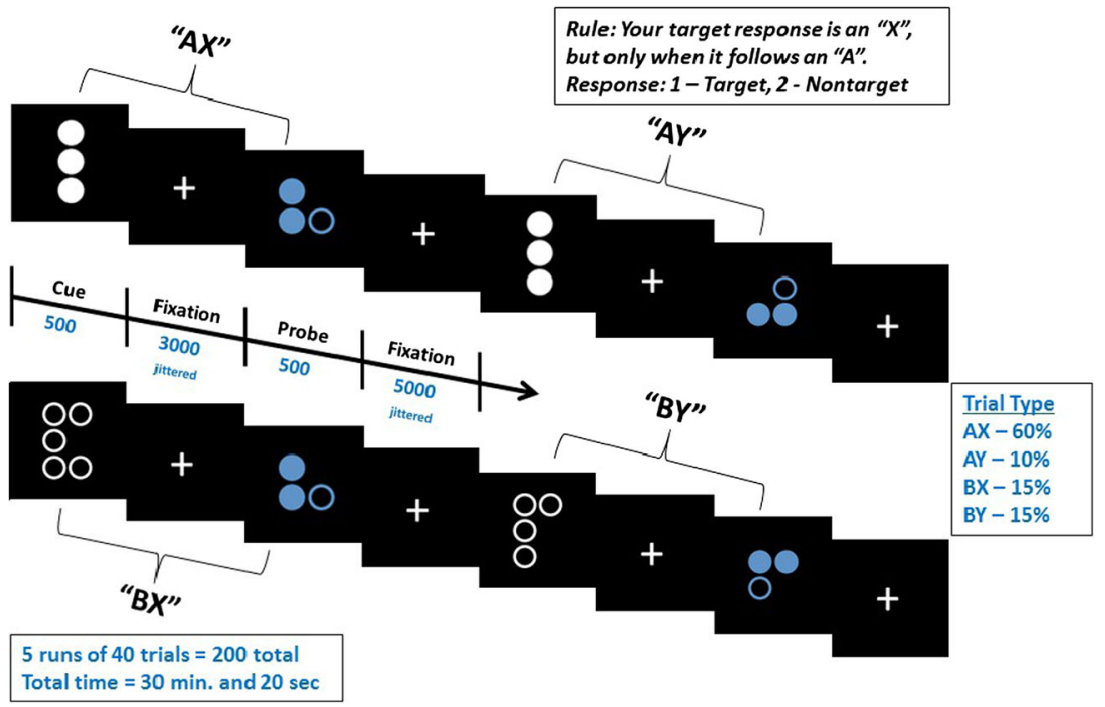

Fig. 1 Task parameters for the AX-CPT and the DPX tasks. Interstimulus intervals were jittered with a mean of 3,000 ms (minimum = 2,200 ms, maximum $=3,500 \mathrm{~ms}$ ), and intertrial intervals were jittered with a mean of 5,000 ms (minimum $=1,700 \mathrm{~ms}$, maximum $=14,500 \mathrm{~ms}$ )

DPX uses unfamiliar Braille figures that would be expected to engage visual-spatial processes) are associated with differences in activation or functional connectivity across the brain.

\section{Method}

\section{Participants}

Twenty-six healthy control subjects were recruited across two sites. No subjects were excluded from the analysis for any reason. Informed consent was obtained from 10 subjects at the Washington University in St. Louis site and 16 subjects at the University of California, Davis. All participants were assessed using the Structured Clinical Interview for the DSMIV-TR [SCID-I/P] and did not meet criteria for any Axis I clinical diagnosis, denied current substance abuse or any substance dependence, screened negative on urine toxicology screens, and were not taking any psychotropic or cognitive enhancing medication. Participants were between the ages of 21 and 43, did not have any clinically significant head injury or neurological disease, had an estimated IQ greater than 70 , 
Table 1 Demographic and behavioral characteristics

\begin{tabular}{ll}
\hline Characteristic & Participants $(n=26)$ \\
\hline & Mean $\pm S D$ \\
Age & $25.61 \pm 5.01$ \\
Sex: $n$ males (\%) & $16(61.5 \%)$ \\
Ethnicity: $n$ Caucasian (\%) & $16(61.5 \%)$ \\
Education: years & $16.5 \pm 1.21$ \\
Behavioral data & \\
AX-CPT accuracy & \\
AX & \\
AY & $.97 \pm .03$ \\
BX & $.9 \pm .09$ \\
BY & $.95 \pm .06$ \\
$d^{\prime}$ context & $.99 \pm .01$ \\
$d^{\prime}$ context & \\
DPX accuracy & $3.82 \pm .73$ \\
AX & $3.51 \pm .72$ \\
AY & \\
BX & $.98 \pm .01$ \\
BY & $.84 \pm .13$ \\
$d^{\prime}$ context & $.92 \pm .08$ \\
$d^{\prime}$ context & \\
AY & $.98 \pm .03$ \\
AX-CPT reaction time & $3.62 \pm .63$ \\
AX & $3.21 \pm .7$ \\
AY & \\
BX & Mean $\pm S D$ \\
BY & $491 \pm 138$ \\
DPX reaction time & $623 \pm 123$ \\
AX & $505 \pm 177$ \\
BY & $495 \pm 157$ \\
\hline
\end{tabular}

and were proficient in English. Table 1 summarizes subjects' demographic characteristics. The study was approved by the Institutional Review Boards at University of California, Davis, and Washington University in St. Louis. Subjects completed the expectancy AX-CPT and DPX tasks during one fMRI session, with the order counterbalanced across subjects. All subjects were paid for their participation.

\section{Task procedure and data analysis}

Participants performed letter (expectancy AX-CPT) and dot pattern (DPX) variants of the CPT in a counterbalanced order. In the letter version of the task subjects are presented with a series of cues and probes. Participants respond to the target sequence $(\mathrm{AX})$ by making an index finger button press to an $\mathrm{X}$ probe only if it was immediately preceded by an A cue. For all other cues and probes, the participants make a middle finger button press. Cues and probes were presented for 500 milliseconds. Interstimulus intervals were jittered with a mean of 3 , $000 \mathrm{~ms}$ (minimum $=2,200 \mathrm{~ms}$, maximum $=3,500 \mathrm{~ms}$ ) and intertrial intervals were jittered with a mean of 5,000 ms (minimum $=1,700 \mathrm{~ms}$, maximum $=14,500 \mathrm{~ms}$ ). Target sequence trials are the most frequent and create a prepotent tendency to make a target response when the target probe occurs. In the dot pattern (DPX) variant, the letters were replaced with dot representations (see Fig. 1). For simplicity, throughout the manuscript, we will refer to the trial types with their "letter" description with the understanding that those letters are actually represented by dot patterns in the DPX.

AX-CPT and DPX accuracy and median reaction times for correct items only were examined using repeated measures analyses of variance (ANOVA) with trial (AX, AY, BX, BY) and task type (AX-CPT and DPX) as main within-subjects factors. Post hoc paired $t$ tests were performed to compare each trial type between tasks. We computed $d^{\prime}$ context as a signal detection index using AX hits and BX false alarms. This measure provides a more specific index of sensitivity to goal maintenance (Cohen et al., 1999; Servan-Schreiber et al., 1996). We also computed a $d^{\prime}$ context $_{\mathrm{AY}}$ score using $\mathrm{AX}$ hits and $\mathrm{AY}$ false alarms. A paired-samples $t$ test was used to compare $d^{\prime}$ context and $d^{\prime}$ context $_{\mathrm{AY}}$ between AX-CPT and DPX.

\section{Functional neuroimaging acquisition and preprocessing}

Task parameters are shown in Fig. 1. Six of the University of California, Davis, participants performed four blocks of each task (rather than five), resulting in 160 trials instead of 200 trials. All task parameters remained the same for these participants. Participants' structural and functional imaging data were collected on $3 \mathrm{~T}$ Siemens TIM Trio scanners at both sites. An agar phantom was used to perform quality assurance of scanners across sites based on guidance and recommendations from the FBIRN initiative. The parameters of the EPI sequence were TR $2000 \mathrm{~ms}$, TE $29 \mathrm{~ms}$, flip angle $75^{\circ}$, FOV $240 \times 240 \mathrm{~mm}$, matrix size $64 \times 64$, with 32 slices and $3.75 \times$ $3.75 \times 4.55 \mathrm{~mm}$ voxels. T1-weighted structural scans and field map scans were also obtained. Each subject's data was preprocessed with Statistical Parametric Mapping-8 software (SPM8, http://www.fil.ion.ucl.ac.uk/SPM8), using all available scans. Slice-timing correction of the functional data was performed, followed by realignment and unwarping. Coregistration of the structural image to the mean functional image was performed, followed by normalization using parameters derived from the normalization of the participant's segmented structural image to the SPM8 gray matter template. Finally, smoothing was performed with an $8 \mathrm{~mm}$ kernel. Individual fMRI runs were removed from the analysis if translational movement exceeded $4 \mathrm{~mm}$ or rotational movement exceeded $3^{\circ}$ of within-run movement, as well as if accuracy 
on any of the trial types was zero. Four runs were excluded for excess within-run movement (two for AX-CPT and two for DPX), and four runs were excluded for not having sufficient correct responses on a given trial type within a run (two for AX-CPT and two for DPX).

Functional imaging analysis was performed in SPM8 by generating a general linear model for each subject with a highpass filter of $75 \mathrm{~s}$. Regressors for both tasks included both cues (A and B cues) and all probes (AX, AY, BX, and BY), as well as error trials. Translational and rotational movement data were included as covariates. We first performed a withintask comparison for the expectancy AX-CPT and the DPX separately through a whole brain voxel-wise analysis, examining the $\mathrm{B}$ cue relative to A cue contrast for each task. Grouplevel random effect comparisons were performed using a twofactor repeated measures ANOVA in SPM8, with task (AX and DPX) as the first factor and cue type (A or B) as the second. Contrasts were generated to examine regions in which greater activity was present in $\mathrm{CueB}$ versus CueA trials for each task as well as regions in which $\mathrm{CueB}$ versus $\mathrm{CueA}$ activity was different between tasks. A covariate was also included to account for site. Whole brain cluster significance was determined by a voxel-level threshold of $p<.005$ and FWE cluster-corrected at $p<.05$. Given the a priori importance of the DLPFC in goal maintenance, ROIs within left and right DLPFC were obtained from a conjunction analysis including both cue types for both tasks. This conjunction map was constrained by masking with bilateral Brodmann Area 9 and 46 masks from the Wake Forest PickAtlas (Maldjian, Laurienti, Burdette, \& Kraft, 2003). Conjunction analyses were performed using the Minimum Statistic compared to the Conjunction Null as described by Nichols and colleagues (Nichols, Brett, Andersson, Wager, \& Poline, 2005).

\section{Connectivity analysis}

The left and right DLPFC ROIs obtained from the conjunction analysis described above were used as seeds in a seed-to-voxel weighted GLM connectivity analysis in the Conn Toolbox (Whitfield-Gabrieli \& Nieto-Castanon, 2012). In each individual subject GLM, the following components were included as confounds: (a) the first five components of the signal from the CSF mask and their first derivatives, (b) main condition effects, and (c) translational and rotational movement parameters and their first derivatives. Band-pass filtering was disabled due to the fast event-related nature of the task design, and detrending and despiking were performed. Each cue was modeled using an HRF-convolved impulse time series (identical to that used in univariate GLM analyses), and positive values for each scan within a particular condition (i.e., $\mathrm{CueB}$ or CueA) were used as weights to compute weighted correlation measures of connectivity between the BOLD time series of each seed with all other voxels in the brain for that trial type.
Connectivity was analyzed using bivariate correlations, and a Fisher transformation (inverse hyperbolic tangent function) was applied to each individual subject correlation map in order to improve normality assumptions. The individual subject seed-to-voxel connectivity maps for the A and $B$ cues for both tasks were included in two separate two-factor repeated measures ANOVAs (one for each seed) in SPM8, with task (AX and DPX) as the first factor and cue (A or $\mathrm{B})$ as the second. Contrasts were generated to examine regions in which greater connectivity was present in $\mathrm{CueB}$ versus CueA trials for each seed. A covariate was also included to account for site. Whole brain cluster significance was determined by a voxel-level threshold of $p<.005$ and FWE cluster-corrected at $p<.05$.

\section{Results}

\section{Behavioral results}

Performance on the expectancy AX-CPT and the DPX tasks are summarized in Table 1 and depicted in Fig. 2.

We conducted a two-way repeated measures ANOVA, which revealed significant main effects of task, $F(1,24)=$ $18.08, p<.001$, due to poorer overall performance in the

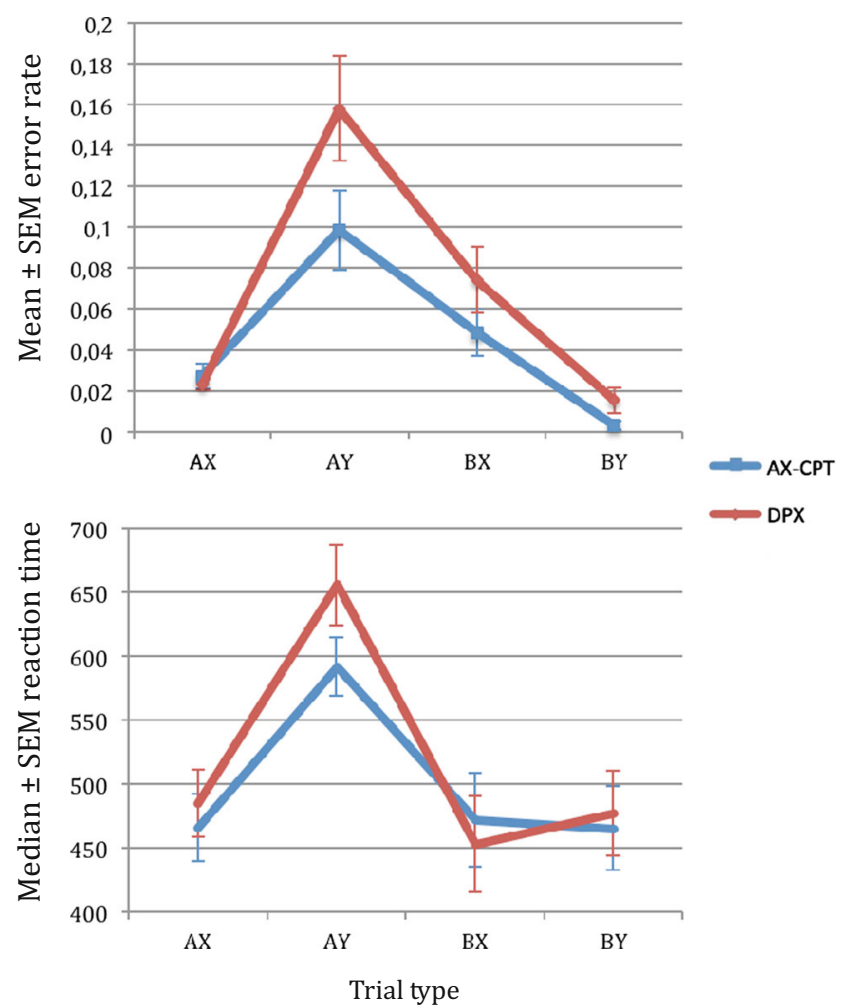

Fig. 2 Accuracy and RT behavioral performance on the expectancy AXCPT and the DPX tasks 
DPX, and of trial type, $F(3,22)=22.25, p<.001$, due to poorer performance on AY trials. The fact that subjects performed worse on AY trials compared to the other trial types in both tasks, reflects the impact of intact context processing leading to increased false alarms during these trials. Finally, there was a trend level significant task by trial type interaction, $\mathrm{F}(3,22)=3.03, p=.072$.

Post hoc contrasts showed significant worse performance on AY trials in DPX compared to AX-CPT, $t=2.72, p=.01$, but not on $\mathrm{AX}, t=-0.72, p=.47$; BX, $t=1.51, p=.14$; and BY trials, $t=1.98, p=.06$. Additionally, the error rate was significantly higher for AY trials compared to BX trials in the AXCPT, $t=2.48, p=.02$, and in the DPX, $t=2.45, p=.02$.

The traditional $d$ 'context score was not significantly different in the expectancy AX-CPT relative to the DPX, $t=1.05$, $p=.29$, although we found differences between tasks in $d^{\prime}$ context $_{\mathrm{AY}}$ calculated as $\mathrm{AX}$ hits versus $\mathrm{AY}$ false alarms, $t=3.61, p<.001$.

In terms of reaction time, there was no main effect of task, $F(1,24)=2.69, p=.113$. However, there was a significant main effect of trial type, $F(3,22)=51.86, p<.001$. There was also a significant interaction between task and trial type, $F(3,22)=5.18, p=.009$.

Post hoc contrasts showed significant slower RT on AY trials in DPX compared to AX-CPT, $t=-2.9, p<.01$; but not on $\mathrm{AX}, t=-1.75, p=.09$; $\mathrm{BX}, t=1.35, p=.19$; and $\mathrm{BY}$ trials, $t=-0.56, p=.58$. AY trials had significantly longer RTs than BX trials in the AX-CPT, $t=6.75, p<.001$, and in the DPX, $t=7.48, p<.001$.

\section{Functional MRI results}

Within-task analysis In the whole brain voxel-wise analysis, we examined the expectancy AX-CPT B-cue relative to A-cue contrast and found widespread activation of the cognitive control network, including bilateral DLPFC, bilateral fusiform gyri, and bilateral inferior parietal cortices (see Table 2 and Fig. 3). In the DPX B-cue versus A-cue contrast, this same fronto-parietal network was significantly activated, including bilateral DLPFC, bilateral fusiform gyri, and right inferior parietal gyrus (see Table 2).

Conjunction analysis The conjunction analysis revealed four clusters of significantly overlapping activity (see Fig. 4), including bilateral DLPFC and bilateral clusters that extended from inferior parietal cortex ventrally to fusiform gyri.

Between-tasks comparison The main differences in activation found for the B-cue versus A-cue contrast when we compared AX-CPT relative to DPX was greater activation for the $\mathrm{AX}-\mathrm{CPT}$ in a cluster extending from the midbrain to left inferior frontal gyrus and bilateral insula (see Fig. 3). Two additional clusters showed greater activity in the AX-CPT, including medial occipital/parietal junction and cerebellum. There were no regions showing greater activation in the DPX as compared to the AX-CPT (see Fig. 3).

\section{Connectivity results}

We did not find any regions that showed differential activity with either the left or right DLPFC seeds for the comparison of B-Cue versus A-Cue trials within either the AX-CPT or the DPX. However, we did find that in a comparison across tasks of connectivity for B-cue versus A-cue trials, the left PFC showed greater functional connectivity with a cluster comprised of the left hippocampus, putamen and midbrain in the DPX compared with the AX-CPT (see Table 3 and Fig. 5). The right PFC also showed increased functional connectivity with three clusters comprised of the left hippocampus/thalamus, putamen/midbrain, and left precentral gyrus in the DPX relative to the AX-CPT (see Fig. 5).

\section{Discussion}

In this study we used a within-subjects design to compare behavioral performance, brain activity and connectivity across both the expectancy AX-CPT and the DPX tasks during fMRI. Behaviorally, participants showed a similar pattern of goal maintenance performance in both tasks although in the DPX there were more errors overall and slower reaction times in the difficult control condition (AY trials). In the analysis of taskrelated activation, both tasks produced robust activation of dorsal frontal and parietal regions associated with goal maintenance, as well as ventral visual regions. Notably, significantly greater activation in elements of this network (i.e., inferior frontal gyrus) and related regions was identified in the expectancy AX-CPT compared to the DPX task. In the functional connectivity analysis, we found greater functional connectivity between prefrontal regions and other brain areas in the DPX compared to the expectancy AX-CPT. In particular, the left PFC showed greater functional connectivity with left hippocampus/putamen, and the right PFC showed greater connectivity with left hippocampus/thalamus, putamen/midbrain, and left precentral gyrus in the DPX compared to the AXCPT.

The expectancy AX-CPT and the DPX have been developed with the goal of reliably and effectively measuring the cognitive and neural systems supporting goal maintenance as operationalized during the CNTRICS and RDoC initiatives. The effectiveness of goal maintenance is evaluated through contrasting performance on BX and AY trials. Participants with preserved goal maintenance show increased AY relative to BX errors and longer AY relative to BX RTs than subjects with impaired goal maintenance (Barch et al., 2003; Cohen et al., 1999; MacDonald, Goghari, et al., 2005; Henderson 
Table 2 Within-group fMRI results for the CueB-CueA contrast for the expectancy AX-CPT and the DPX tasks

\begin{tabular}{|c|c|c|c|c|c|c|c|}
\hline \multirow[t]{2}{*}{ Region of Activation $^{+}$} & \multirow[t]{2}{*}{$\mathrm{BA}$} & \multirow[t]{2}{*}{$\mathrm{R} / \mathrm{L}$} & \multirow[t]{2}{*}{ Size $\left(\mathrm{mm}^{3}\right)$} & \multirow[t]{2}{*}{ Peak $T$ value } & \multicolumn{3}{|c|}{ MNI coordinates } \\
\hline & & & & & $x$ & $y$ & $z$ \\
\hline \multicolumn{8}{|l|}{$A X$} \\
\hline Middle Frontal Gyrus & 9 & $\mathrm{R}$ & 90,320 & 7,45 & 48 & 12 & 26 \\
\hline Insula & & $\mathrm{R}$ & & 6,35 & 32 & 22 & 0 \\
\hline Supplementary Motor Area & 6 & $\mathrm{~L}$ & & 4,86 & -16 & -2 & 64 \\
\hline Fusiform Gyrus & & $\mathrm{R}$ & 209,184 & 7,31 & 44 & -50 & -18 \\
\hline Inferior Parietal Cortex & 40 & $\mathrm{R}$ & & 6.10 & 30 & -68 & 44 \\
\hline Fusiform Gyrus & 37 & $\mathrm{~L}$ & & 7,05 & -40 & -54 & -14 \\
\hline Inferior Parietal Gyrus & 40 & $\mathrm{~L}$ & & 5,84 & -26 & -70 & 40 \\
\hline Middle Frontal Gyrus & 9 & $\mathrm{~L}$ & 38,912 & 6,01 & -46 & 4 & 26 \\
\hline \multicolumn{8}{|l|}{$D P X$} \\
\hline Fusiform Gyrus & 37 & $\mathrm{R}$ & 54,728 & 7.4 & 40 & -80 & -8 \\
\hline Inferior Parietal Cortex & 40 & $\mathrm{R}$ & & 5.63 & 28 & -64 & 38 \\
\hline Fusiform Gyrus & 37 & $\mathrm{~L}$ & 35,776 & 6,71 & -34 & -84 & -8 \\
\hline Inferior Parietal Cortex & 39 & $\mathrm{~L}$ & & 3,84 & -22 & -72 & 54 \\
\hline Middle Frontal Gyrus & 9 & $\mathrm{R}$ & 12,296 & 5,17 & 52 & 14 & 30 \\
\hline Middle Frontal Gyrus & 9 & $\mathrm{~L}$ & 7,264 & 4,17 & -56 & 8 & 32 \\
\hline \multicolumn{8}{|l|}{ AX greater than $D P X$} \\
\hline Inferior Frontal Gyrus & 47 & $\mathrm{~L}$ & 9,600 & 4,15 & -42 & 20 & 0 \\
\hline Insula & & $\mathrm{L}$ & & 3.90 & -32 & 6 & 2 \\
\hline Putamen & & $\mathrm{L}$ & & 3.31 & -30 & 12 & 0 \\
\hline Globus Pallidus & & $\mathrm{L}$ & & 3.33 & -20 & -6 & 0 \\
\hline Midbrain & & $\mathrm{L}$ & & 3.72 & -10 & -12 & -8 \\
\hline Globus Pallidus & & $\mathrm{R}$ & & 3.29 & 20 & -8 & -6 \\
\hline Cerebellum & & $\mathrm{L}$ & 4,424 & 4,14 & -2 & -48 & -16 \\
\hline Cerebellum & & $\mathrm{R}$ & & 3.60 & 6 & -50 & -6 \\
\hline Lingual Gyrus & 19 & $\mathrm{R}$ & & 3.17 & 6 & -64 & -4 \\
\hline Insula & & $\mathrm{R}$ & 4,312 & 3.89 & 40 & 14 & 4 \\
\hline Cuneus & 31 & $\mathrm{~L}$ & 4,104 & 3.02 & -10 & -76 & 24 \\
\hline Precuneus & 31 & $\mathrm{~L}$ & & 3,12 & -4 & -76 & 24 \\
\hline Precuneus & 31 & $\mathrm{R}$ & & 3,08 & 6 & -76 & 20 \\
\hline Cuneus & 31 & $\mathrm{R}$ & & 3,21 & 10 & -74 & 30 \\
\hline \multicolumn{8}{|l|}{$D P X$ greater than $A X$} \\
\hline No significant clusters & & & & & & & \\
\hline
\end{tabular}

+Large clusters were identified as significant within each task. Consequently local maxima are also listed underneath each significant cluster and identified by an indent

BA, Brodmann Area; R, Right; L, Left; MNI, Montreal Neurological Institute

et al., 2012; Jones et al., 2010). Our behavioral results in a sample of healthy control subjects show a pattern of intact goal maintenance. There was increase in the number of AY errors relative to BX errors in both tasks, as well as slower RTs on AY trials as compared to BX trials. These results are consistent with the extensive literature on goal maintenance measured through the AX-CPT/DPX (Barch et al., 2001; Braver et al., 1999; Braver \& Cohen, 1999; Cohen \& ServanSchreiber, 1993).
In the between-task comparison, we found that participants had significantly lower accuracy and slower RTs on AY trials on the DPX compared to the expectancy AX-CPT. This is likely due to the increased demands during the DPX in assigning task-related meaning ("decoding") to the underlearned visual renderings of Braille stimuli used in this task, and there are a number of mechanisms that could underlie this effect. One possible account is that the increased attentional demands (or increased cognitive load) required for the 

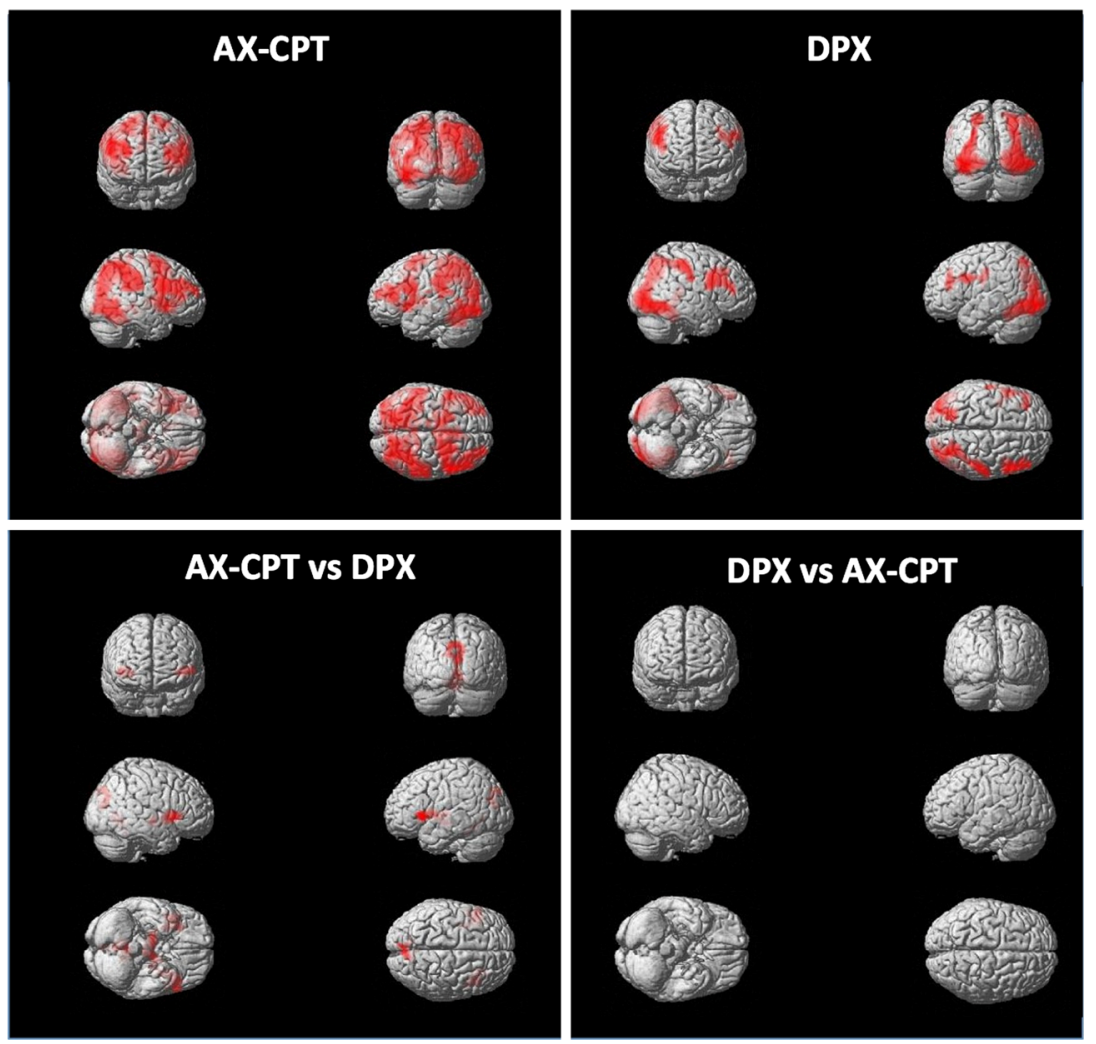

Fig. 3 Within-group univariate fMRI results for the CueB-CueA contrast in the expectancy AX-CPT, DPX task, and between-task comparisons (voxel-level threshold of $p<.005$ and FWE cluster-

corrected at $p<.05)$. Images are displayed according to neurological convention (left side of the image corresponds to the left side of the brain)

DPX may lead to a more robust context representation being maintained during the cue probe interval. This increase in attentional demands during the performance of the DPX would result in slower performance and higher error rates in all trial types and specifically poorer performance on AY trials due to an increase in the context representation of the A cue. This would lead to the development of stronger expectancies and more false alarms in the AY trials in healthy subjects as they prepare to make a target response following the occurrence of the A cue. The connectivity with clusters including the hippocampus/thalamus and striatum suggests that this decoding process may involve the use of long term memory representations, and the need to use these representations may come with a cost to performance. We are unable to adjudicate as to which of these possible accounts, or a combination of them, underlies the increase in AY errors on the DPX, however it is likely that this effect is linked to processes involved in goal maintenance given the selective differences in the AY condition. If it were due to an overall increase in perceptual difficulty associated with the DPX, we would have expected poorer performance on all trials compared to the letter version of the task (expectancy AX-CPT) rather than being selectively worse in the AY condition.
One of the reasons for the selection by the CNTRICS initiative of the expectancy AX-CPT and DPX to study goal maintenance was the evidence that the AX-CPT tapped the neural systems relevant for the construct of goal maintenance (Barch, Moore, Nee, Manoach, \& Luck, 2012). To our knowledge, there are no published neuroimaging studies to date of the DPX. Numerous studies have used the AX-CPT to examine the neural substrate of goal maintenance in healthy and in clinical populations (Barch et al., 2001; Holmes et al., 2005; Lesh et al., 2013; MacDonald \& Carter 2003; MacDonald et al., 2003; MacDonald, Carter, et al., 2005; Paxton et al., 2008; Perlstein et al., 2001; Yoon et al., 2008). Such studies have repeatedly found activation in the dorsolateral regions of PFC (BA 46 and 9), anterior cingulate and inferior parietal cortex during demands on goal maintenance. It is thought that the engagement of DLPFC during goal maintenance is necessary for the performance of the task, regardless of task modality (MacDonald \& Carter, 2003). However, DLPFC may provide top-down input to other regions that might be more sensitive to modality (Fuster, Bodner, \& Kroger, 2000; Miller, 2000), such as the differing verbal, visuospatial processing, and perceptual learning requirements required by the AX-CPT versus the DPX. We found a qualitatively similar pattern of 
Table 3 Functional connectivity analysis of AX-CPT and the DPX tasks (FWE cluster corrected $\mathrm{p}<0.05$ )

\begin{tabular}{|c|c|c|c|c|c|c|c|}
\hline \multirow[t]{2}{*}{ Region of Activation } & \multirow[t]{2}{*}{$\mathrm{BA}$} & \multirow[t]{2}{*}{$\mathrm{L} / \mathrm{R}$} & \multirow[t]{2}{*}{ Size $\left(\mathrm{mm}^{3}\right)$} & \multirow[t]{2}{*}{ Peak $T$ value } & \multicolumn{3}{|c|}{ MNI coordinates } \\
\hline & & & & & $x$ & $y$ & $z$ \\
\hline \multicolumn{8}{|l|}{ Left PFC seed } \\
\hline \multicolumn{8}{|l|}{$A X$} \\
\hline \multicolumn{8}{|l|}{ No significant clusters } \\
\hline \multicolumn{8}{|l|}{$D P X$} \\
\hline \multicolumn{8}{|l|}{ No significant clusters } \\
\hline \multicolumn{8}{|l|}{$A X$ greater than $D P X$} \\
\hline \multicolumn{8}{|l|}{ No significant clusters } \\
\hline \multicolumn{8}{|l|}{$D P X$ greater than $A X$} \\
\hline Hippocampus/ PHG & & $\mathrm{L}$ & 17,360 & 5.15 & -30 & -18 & -12 \\
\hline Putamen & & $\mathrm{L}$ & & 4.07 & -24 & 2 & 10 \\
\hline Putamen & & $\mathrm{L}$ & & 4.07 & -18 & 10 & -8 \\
\hline Midbrain & & $\mathrm{L}$ & & 3.45 & -8 & -14 & -12 \\
\hline Midbrain & & I & & 3.89 & 0 & -32 & -16 \\
\hline Midbrain & & $\mathrm{R}$ & & 3.98 & 6 & -10 & -14 \\
\hline Globus Pallidus & & $\mathrm{R}$ & & 3.57 & 6 & 0 & -6 \\
\hline Subgenual Cingulate & 25 & $\mathrm{R}$ & & 3.44 & 16 & 22 & -10 \\
\hline \multicolumn{8}{|l|}{ Right PFC seed } \\
\hline \multicolumn{8}{|l|}{$A X$} \\
\hline \multicolumn{8}{|l|}{ No significant clusters } \\
\hline \multicolumn{8}{|l|}{$D P X$} \\
\hline \multicolumn{8}{|l|}{ No significant clusters } \\
\hline \multicolumn{8}{|l|}{$A X$ greater than $D P X$} \\
\hline \multicolumn{8}{|l|}{ No significant clusters } \\
\hline \multicolumn{8}{|l|}{$D P X$ greater than $A X$} \\
\hline Anterior Hippocampus & & $\mathrm{L}$ & 6,584 & 4.02 & -32 & -6 & -22 \\
\hline Thalamus & & $\mathrm{L}$ & & 4.32 & -22 & -22 & 8 \\
\hline Middle Hippocampus & & $\mathrm{L}$ & & 3.93 & -30 & -14 & -10 \\
\hline Thalamus & & $\mathrm{L}$ & & 3.85 & -14 & -16 & 6 \\
\hline Putamen & & $\mathrm{L}$ & 11,600 & 4.31 & -18 & 12 & -6 \\
\hline Midbrain & & $\mathrm{R}$ & & 3.88 & 2 & -22 & -16 \\
\hline Midbrain & & $\mathrm{R}$ & & 4.37 & 12 & -12 & -14 \\
\hline Putamen & & $\mathrm{R}$ & & 3.87 & 16 & 2 & -12 \\
\hline Precentral Gyrus & 6 & $\mathrm{~L}$ & 5,880 & 4.17 & -38 & -6 & 64 \\
\hline Precentral Gyrus & 6 & $\mathrm{~L}$ & & 3.10 & -42 & -22 & 68 \\
\hline Postcentral Gyrus & 3 & $\mathrm{~L}$ & & 3.80 & -30 & -30 & 74 \\
\hline Superior Frontal Gyrus & 6 & $\mathrm{~L}$ & & 3.71 & -26 & -8 & 74 \\
\hline Postcentral Gyrus & 3 & $\mathrm{~L}$ & & 2.96 & -22 & -28 & 78 \\
\hline Precentral Gyrus & 6 & $\mathrm{~L}$ & & 4.02 & -10 & -14 & 80 \\
\hline Superior Frontal Gyrus & 6 & $\mathrm{R}$ & & 3.87 & 14 & -4 & 80 \\
\hline
\end{tabular}

BA, Brodmann Area; R, Right; L, Left; MNI, Montreal Neurological Institute; PFC, Prefrontal Cortex; PHG, Parahippocampal Gyrus

activations across both tasks, which including activation in bilateral DLPFC, right inferior parietal gyrus, and bilateral fusiform gyrus. However, in the direct comparison between both tasks, we did see evidence of stronger activations in the
AX-CPT compared to the DPX, including significant differences in the frontal opercular insula. Although there are a number of processes associated to the activation of the insula, this difference may be consistent with subjects' greater 


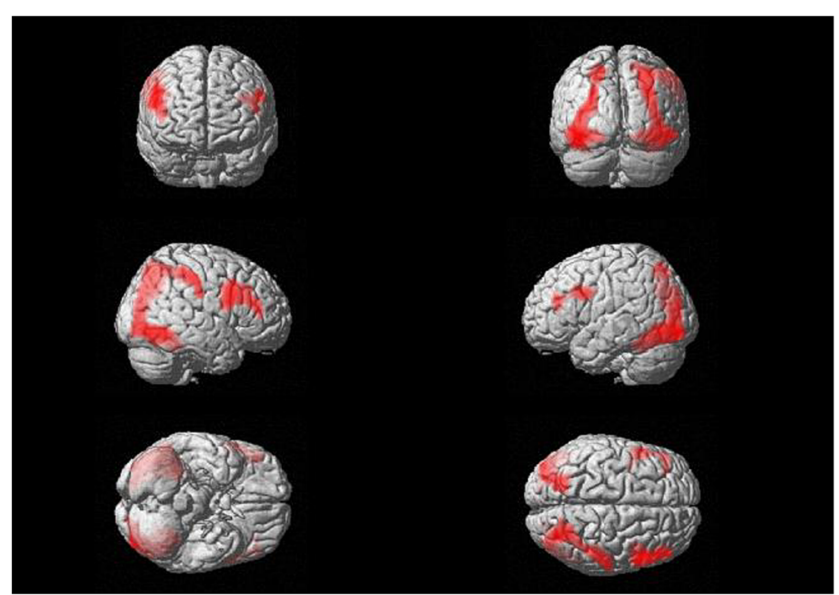

Fig. 4 The conjunction analysis revealed four clusters of significantly overlapping activity, including bilateral DLPFC and bilateral clusters that extended from inferior parietal cortex ventrally to fusiform gyri

reliance on phonological strategies during encoding and maintenance of the cue on the letter version of the task.
In terms of functional connectivity, we identified a number of significant differences between the tasks, the most robust being greater connectivity between the PFC and a cluster comprising the hippocampus and thalamus during DPX compared to the AX-CPT for B- versus A-cue trials. B-cue trials reflect the ability to use goal representations when a nontarget response has to be made and when the goal information represented by the cue has to be used to overcome a prepotent response tendency. The DLPFC is a region that supports the representation and active maintenance of stimuli to guide response selection toward a goal and therefore is expected to be engaged during decisions that require context processing (Miller \& Cohen 2001). In the AX-CPT, the familiar letter stimuli and the responses associated with them are easily stored in working memory. The novel dot configurations in the DPX may require additional effort to encode and retrieve the task appropriate stimulus response mappings from longterm memory during each stimulus presentation. This may lead to increased engagement of brain systems related to spatial processing and memory. The medial temporal cortical

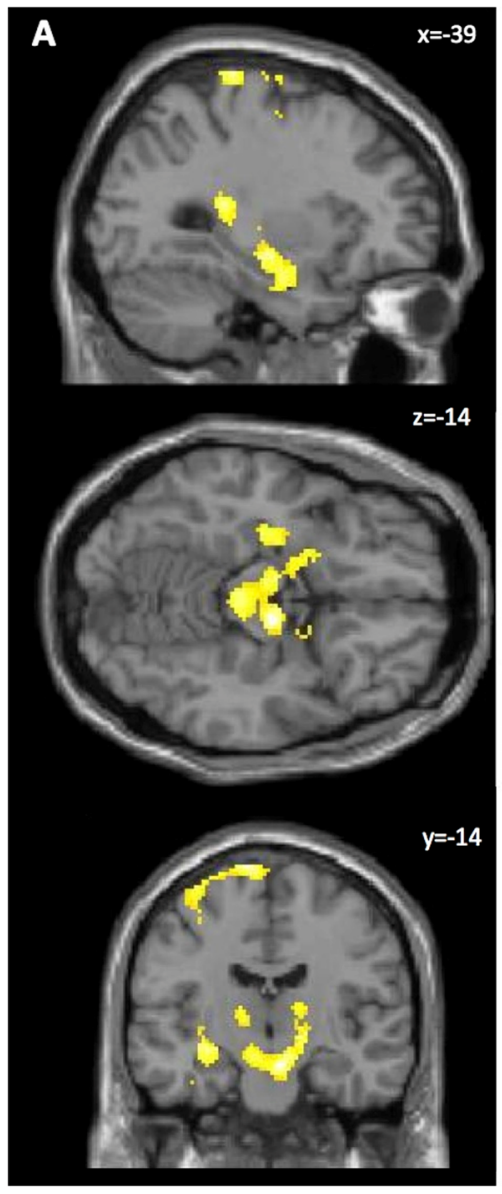

Fig. 5 Functional connectivity in which DPX shows greater connectivity between right PFC seed and left hippocampus, striatum and thalamus (a) and greater connectivity between left PFC and left hippocampus (b) relative to the AX-CPT (voxel-level threshold of $p<.005$ and FWE

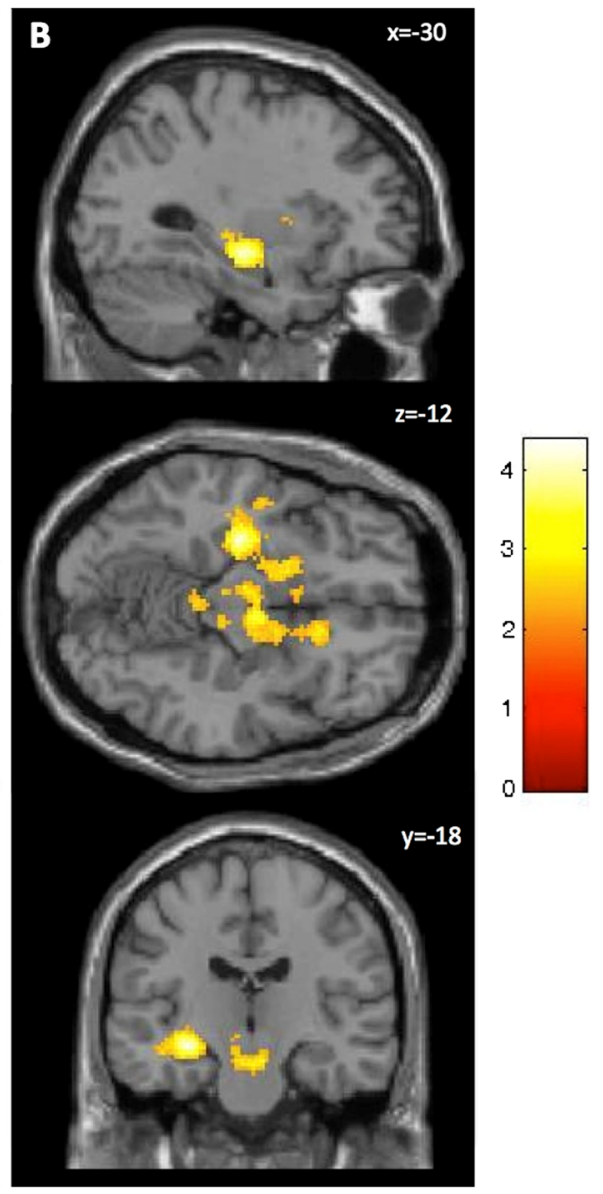

cluster-corrected at $p<.05)$. Images are displayed according to neurological convention (left side of the image corresponds to the left side of the brain) 
regions seem to be involved in maintaining representations of spatial stimuli (Bird \& Burgess, 2008) and of novel and complex objects (Ranganath \& Blummenfeld, 2005), providing feedback to prefrontal areas in order to guide working memory maintenance (Ranganath, 2006). While we did not see activation in the medial temporal lobe during the DPX, the selective increase of connectivity between DLPFC and this region seen during the performance of the DPX compared to the AX-CPT suggests that the stimulus characteristics of the DPX elicit hippocampus-prefrontal cortex connectivity while the highly familiar stimuli of the AX-CPT engage mainly prefrontal areas during goal-maintenance processes. Along with the hippocampus, in the comparison between DPX and AX-CPT we found greater connectivity between frontal areas and putamen/midbrain. Taken together, these areas are part of a large network involved in spatial attention (Kim et al., 1999), which seems to be elicited during the DPX, requiring greater spatial attention resources.

These connectivity results complement those of the univariate analysis in suggesting that the basic engagement of the cognitive control network is similar during both versions of the CPT, but differences in the stimulus characteristics of the cue are also associated with greater engagement of systems related to phonological processing in the expectancy AX and with visual-spatial processing (and possibly long-term memory) in the DPX task.

The use of paradigms from cognitive neuroscience such as the AX-CPT and the DPX in translational research may serve as sensitive measures of the specific cognitive and neural systems implicated in schizophrenia and related disorders. We have studied the neural circuitry supporting goal maintenance during cognitive control, an important area of impairment in schizophrenia (Barch et al., 2003; Barch \& Braver 2005; Braver et al., 1999; Cohen et al., 1999; Lesh et al., 2013; MacDonald et al., 2003). Our findings support the use of both tasks in studying goal maintenance impairment and its neural correlates in clinical populations.

\section{General conclusion}

In this study, we have compared two measures of context processing, the expectancy AX-CPT and the DPX tasks, to investigate the functional activity and functional connectivity engaged during the performance of both tasks. We found that both tasks engage the same brain network, including DLPFC, fusiform, and parietal areas, supporting their construct validity as complementary measures of the goal maintenance construct. Greater engagement of the frontal opercular and anterior insula during the expectancy $\mathrm{AX}$ and greater functional connectivity between the PFC and medial temporal lobe, thalamus, and striatum in the DPX are consistent with differential recruitment of phonological and visual-spatial networks across the two tasks, and it is also possible that the underlearned nature of the dot probe stimuli may engage additional long term memory systems not engaged by the expectancy version of the task.

\section{References}

Barch, D.M., Berman, M.G., Engle, R., Hurdelbrink Jones, J., Jonides, J., MacDonald, A. III, . . . Sponheim, S.R. (2009). CNTRICS final task selection: Working memory. Schizophrenia Bulletin, 35, 136-152.

Barch, D. M., \& Braver, T. S. (2005). Cognitive control in schizophrenia: Cognitive and neural mechanisms. In R. W. Engle, G. Sedek, U. von Hecker, \& D. N. McIntosh (Eds.), Cognitive limitations in aging and psychopathology: Attention, working memory, and executive functions (pp. 122-159). Cambridge: Cambridge University Press.

Barch, D. M., Braver, T. S., Nystom, L. E., Forman, S. D., Noll, D. C., \& Cohen, J. D. (1997). Dissociating working memory from task difficulty in human prefrontal cortex. Neuropsychologia, 35, 13731380.

Barch, D. M., Carter, C. S., Braver, T. S., McDonald, A., Sabb, F. W., Noll, D. C., \& Cohen, J. D. (2001). Selective deficits in prefrontal cortex function in medication-naive patients with schizophrenia. Archives of General Psychiatry, 58, 280-288.

Barch, D. M., Carter, C. S., Dakin, S. C., Gold, J., Luck, S. J., MacDonald, A., III, \& Strauss, M. E. (2012). The clinical translation of a measure of gain control: The contrast-contrast effect task. Schizophrenia Bulletin, 38, 135-143.

Barch, D. M., Carter, C. S., MacDonald, A. W., III, Braver, T. S., \& Cohen, J. D. (2003). Context-processing deficits in schizophrenia: Diagnostic specificity, 4-week course, and relationships to clinical symptoms. Journal of Abnormal Psychology, 112(1), 132-143.

Barch, D. M., \& Smith, E. (2008). The cognitive neuroscience of working memory: Relevance to CNTRICS and schizophrenia. Biological Psychiatry, 64, 11-17.

Bird, C. M., \& Burgess, N. (2008). The hippocampus and memory: Insights from spatial processing. Nature Reviews Neuroscience, 9(3), 182-194.

Braver, T. S., Barch, D. M., \& Cohen, J. D. (1999). Cognition and control in schizophrenia: A computational model of dopamine and prefrontal function. Biological Psychiatry, 46, 312-328.

Braver, T. S., \& Cohen, J. D. (1999). Dopamine, cognitive control, and schizophrenia: The gating model. Progress in Brain Research, 121, 327-349.

Carter, C. S., \& Barch, D. M. (2007). Cognitive neuroscience-based approaches to measuring and improving treatment effects on cognition in schizophrenia: The CNTRICS initiative. Schizophrenia Bulletin, 33, 1131-1137.

Carter, C.S., Barch, D.M., Buchanan, R.W., Bullmore, E., Krystal, J.H., Cohen, J., . . . Heinssen, R. (2008). Identifying cognitive mechanisms targeted for treatment development in schizophrenia: An overview of the first meeting of the cognitive neuroscience treatment research to improve cognition in schizophrenia initiative. Biological Psychiatry, 64, 4-10.

Cohen, J. D., Barch, D. M., Carter, C., \& Servan-Schreiber, D. (1999). Context-processing deficits in schizophrenia: Converging evidence from three theoretically motivated cognitive tasks. Journal of Abnormal Psychology, 108(1), 120-133.

Cohen, J. D., \& Servan-Schreiber, D. (1993). A theory of dopamine function and its role in cognitive deficits in schizophrenia. Schizophrenia Bulletin, 19(1), 85-104. 
Fuster, J. M., Bodner, M., \& Kroger, J. K. (2000). Cross-modality and cross-temporal associations in neurons of frontal cortex. Nature, 405, 347-351.

Henderson, D., Poppe, A., Barch, D.M., Carter, C.S., Gold, J.M., Ragland, J. D., . . MacDonald, A.W. III. (2012). Optimization of a goal maintenance task for use in clinical applications. Schizophrenia Bulletin, 38, 104-113.

Holmes, A. J., MacDonald, A., III, Carter, C. S., Barch, D. M., Stenger, V. A., \& Cohen, J. D. (2005). Prefrontal functioning during context processing in schizophrenia and major depression: An eventrelated fMRI study. Schizophrenia Research, 76, 199-206.

Javitt, D. C., Shelley, A. M., Silipo, G., \& Lieberman, J. A. (2000). Deficits in auditory and visual context-dependent processing in schizophrenia. Archives of General Psychiatry, 57, 1131-1137.

Jones, J. A., Sponheim, S. R., \& MacDonald, A. W., III. (2010). The dot pattern expectancy task: Reliability and replication of deficits in schizophrenia. Psychological Assessment, 22(1), 131-141.

Kim, Y. H., Gitelman, D. R., Nobre, A. C., Parrish, T. B., LaBar, K. S., \& Mesulam, M. (1999). The large-scale neural network for spatial attention displays multifunctional overlap but differential asymmetry. NeuroImage, 9(3), 269-277.

Lesh, T. A., Westphal, A. J., Niendam, T. A., Yoon, J. H., Minzenberg, M. J., Ragland, J. D., \& Carter, C. S. (2013). Proactive and reactive cognitive control and dorsolateral prefrontal cortex dysfunction in first episode schizophrenia. NeuroImage Clinical, 2, 590-599.

Lopez-Garcia, P., Young Espinoza, L., Molero Santos, P., Marin, J., \& Ortuño Sanchez-Pedreño, F. (2013). Impact of COMT genotype on cognition in schizophrenia spectrum patients and their relatives. Psychiatry Research, 208(2), 118-124.

MacDonald, A. W., III. (2008). Building a clinically-relevant cognitive task: Case study of the AX paradigm. Schizophrenia Bulletin, 34, 619-628.

MacDonald, A.W. III, Carter, C.S., Kerns, J.G., Ursu, S., Barch, D.M., Holmes, B. A., . . . Cohen, J.D. (2005). Specificity of prefrontal dysfunction and context processing deficits to schizophrenia in a never medicated first-episode psychotic sample. American Journal of Psychiatry, 162, 475-484.

MacDonald, A. W., III, \& Carter, C. S. (2003). Event-related fMRI study of context processing in dorsolateral prefrontal cortex of patients with schizophrenia. Journal of Abnormal Psychology, 112, 689697.

MacDonald, A. W., Goghari, V. M., Hicks, B. M., Flory, J. D., Carter, C. S., \& Manuck, S. B. (2005). A convergent-divergent approach to context processing, general intellectual functioning, and the genetic liability to schizophrenia. Neuropsychology, 19(6), 814-821.

MacDonald, A. W., III, Pogue-Geile, M. F., Johnson, M. K., \& Carter, C. S. (2003). A specific deficit in context processing in the unaffected siblings of patients with schizophrenia. Archives of General Psychiatry, 60(1), 57-65.
Maldjian, J. A., Laurienti, P. J., Burdette, J. B., \& Kraft, R. A. (2003). An automated method for neuroanatomic and cytoarchitectonic atlasbased interrogation of fMRI data sets. NeuroImage, 19, 1233-1239.

Miller, E. K. (2000). The prefrontal cortex and cognitive control. Nature Reviews, 1, 59-65.

Miller, E. K., \& Cohen, J. D. (2001). An integrative theory of prefrontal cortex function. Annual Review of Neuroscience, 24, 167-202.

Niendam, T.A., Lesh, T.A., Yoon, J., Westphal, A.J., Hutchison, N., Ragland, D., . . . Carter, C.S. (2014). Impaired context processing as a potential marker of psychosis risk state. Psychiatry Research, 221(1), 13-20.

Nichols, T., Brett, M., Andersson, J., Wager, T., \& Poline, J. B. (2005). Valid conjunction inference with the minimum statistic. Neuroimage, 25(3), 653-60.

Paxton, J. L., Barch, D. M., Racine, C. A., \& Braver, T. S. (2008). Cognitive control, goal maintenance and prefrontal function in healthy aging. Cerebral Cortex, 18, 1010-1028.

Perlstein, W. M., Carter, C. S., Noll, D. C., \& Cohen, J. D. (2001). Relation of prefrontal cortex dysfunction to working memory and symptoms of schizophrenia. American Journal of Psychiatry, 158, $1105-1113$.

Ranganath, C. (2006). Working memory for visual objects: Complementary roles of inferior temporal, medial temporal, and prefrontal cortex. Neuroscience, 139, 277-289.

Ranganath, C., \& Blumenfeld, R. S. (2005). Doubts about double dissociations between short- and long-term memory. Trends in Cognitive Science, 9(8), 374-380.

Rosvold, K. E., Mirsky, A. F., Sarason, I., Bransome, E. D., \& Beck, L. H. (1956). A continuous performance test of brain damage. Journal of Consulting Psychology, 20, 343-350.

Servan-Schreiber, D., Cohen, J. D., \& Steingard, S. (1996). Schizophrenic deficits in the processing of context. A test of a theoretical model. Archives of General Psychiatry, 53, 1105-1112.

Whitfield-Gabrieli, S., \& Nieto-Castanon, A. (2012). Conn: A functional connectivity toolbox for correlated and anticorrelated brain networks. Brain Connectivity, 2(3), 125-141.

Yoon, J. H., Minzenberg, M. J., Ursu, S., Walter, R., Wendelken, C., Ragland, J. D., \& Carter, C. S. (2008). Association of dorsolateral prefrontal cortex dysfunction with disrupted coordinated brain activity in schizophrenia: Relationship with impaired cognition, behavioral disorganization, and global function. American Journal of Psychiatry, 165(8), 1006-1014.

Zhang, Q., Shen, Q., Xu, Z., Chen, M., Cheng, L., Zhai, J., . . Chen, C. (2012). The effects of CACNA1C gene polymorphism on spatial working memory in both healthy controls and patients with schizophrenia or bipolar disorder. Neuropsychopharmacology, 37(3), 677-84.

Zhu, X., Gu, H., Liu, Z., Xu, Z., Chen, X., Sun, X., . . . Chen, C. (2013). Associations between TCF4 gene polymorphism and cognitive functions in schizophrenia patients and healthy controls. Neuropsychopharmacology, 38(4), 683-689. 